\title{
Model-Based Localization Method by Non-speech Sound Via Wavelet Transform and Dynamic Neural Network
}

\author{
Albert Marzàbal, Antoni Grau, and Yolanda Bolea \\ Automatic Control Dept, Technical University of Catalonia UPC, \\ c/ Pau Gargallo 5, 08028 Barcelona, Catalonia, Spain \\ \{albert.marzabal, antoni.grau, yolanda.bolea\}@upc.edu
}

\begin{abstract}
Non-speech sound processing is of great interest for indoor mobile robot localization. This paper presents a technique based on feature extraction from continuous wavelet transform (CWT) and a dynamic feed-forward neural network that will approximate the position of the robot in the spatial domain. The link between the function approximation stage (ANN) and the feature extraction stage (CWT) is performed by feature comparative analysis.
\end{abstract}

\section{Introduction}

The reverberation effects in a closed room depend mainly on the walls characteristics -such as paralelism, distances and absortion coefficient- and, to a lesser extent, on the objects characteristics located in the room.

Due to reverberations, a sound source located in a specific and known point in the room -a milling machine for instance- characterizes the sound register acquired in different points in the room for the effects of signal bounces feedforward and cancellation. Since the reverberation effect in the temporal composition of the samples depends on the distance and the signal wavelength, a register composed with different frequencies will also contain information of reverberations in different frequencies. Based on this effect, this work studies the use of non-speech sound information to develop a model-based localization method for mobile robots.

Huang et al. 4 proposed a sound localization method based on a model of human auditory to cope with echoes and reverberations, performing a psychological research over human listener skills, and they propose an acustical preprocess for inhibite input sound when the composition of echoes and reverberations is high. By the other hand, Cowling \& Citte [5] presented a comparative study for different techniques that are tipically used in music recognition. They showed that the use of continuous wavelet transform yields good classification rates. In 6], the use of neural networks to sound recognition demostrates to be a useful tool to achieve high rates in patterns identification and recognition.

In a previous work, Bolea et al. [1] have also dealt with non-speech sound localization using wavelet transform and proposed a polynomical transformation 
from the feature domain to the spatial domain. The work presented interesting results but it did not cover the non-linealities that sound reverberation presents. For this reason, in this work we collect a set of sound registers that will be classified with a dynamic (feed-forward) neural network in the bidimensional space (Section 4). In 2] the use of continuous wavelet transform has been applied to recognition of industrial sound sources, and [3] also uses wavelet transform to detect defects in laser welding by this industrial sound.

In this work we proposed the use of the continuous wavelet transform (Section 2) for its great capability of characterize the information contained in the sound signal. In Section 3 the feature extraction from the wavelet coefficients is presented, and some interesting experiments with a mobile robot are shown in Section [5.

In Figure 1 the complet procedure is shown. Firstly, the sound is obtained with an ommidirectional microphone in a room (later, in Section 5 there is a description of how the sound is acquired). The signal, digitally sampled and coped, is entered to the wavelet transform block. The output of this stage are the coeffcients of the CWT which are used as input for the feature extraction stage that reduces largely the amount of data and prepares the input for the neural network. There are two steps: the learning step and the recognition step. The network is trained with a huge number of sound samples and it is validated with another subset of samples. In the following sections, the different stages are explained.

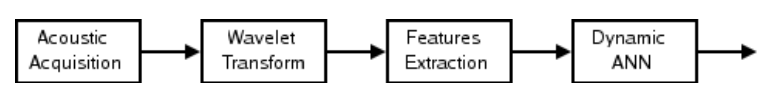

Fig. 1. Localization by non-speech sound features process

\section{Continuous Wavelet Transform}

Wavelet analysis is similar to Fourier analysis in the sense that it breaks a signal down into its constituent parts for analysis. Whereas the Fourier transform breaks the signal into a series of sine waves of different frequencies, the wavelet transform breaks the signal into its "wavelets", scaled and shifted versions of the "mother wavelet".

When analysing signals of a non-stationary nature, it is often beneficial to be able to acquire a correlation between the time and frequency domains of a signal. The Fourier transform, provides information about the frequency domain, however localised time information is essentially lost in the process. The problem with this is the inability to associate features in the frequency domain with their location in time, as an alteration in the frequency spectrum will result in changes throughout the time domain. In contrast to the Fourier transform, the wavelet transform allows exceptional localisation in both the time domain via translations of the mother wavelet, and in the scale (frequency) domain via dilations. The translation and dilation operations applied to the mother 
wavelet are performed to calculate the wavelet coefficients, which represent the correlation between the wavelet and a localised section of the signal. The wavelet coefficients are calculated for each wavelet segment, giving a time-scale function relating the wavelets correlation to the signal.

We used the Continuous Wavelet Transform algorithm (figure 2). Here * denotes complex conjugate

$$
C W T_{(a, b)}=\frac{1}{\sqrt{|a|}} \int_{-\infty}^{+\infty} \psi_{\left(\frac{t-b}{a}\right)}^{*} \cdot f_{(x)} d x
$$

where $b$ represents translation, $a$ represents scale and $\psi_{(t)}$ is the Morlet mother wavelet defined as:

$$
\psi_{(t)}=e^{j a t} e^{\frac{-t^{2}}{2 s}}
$$
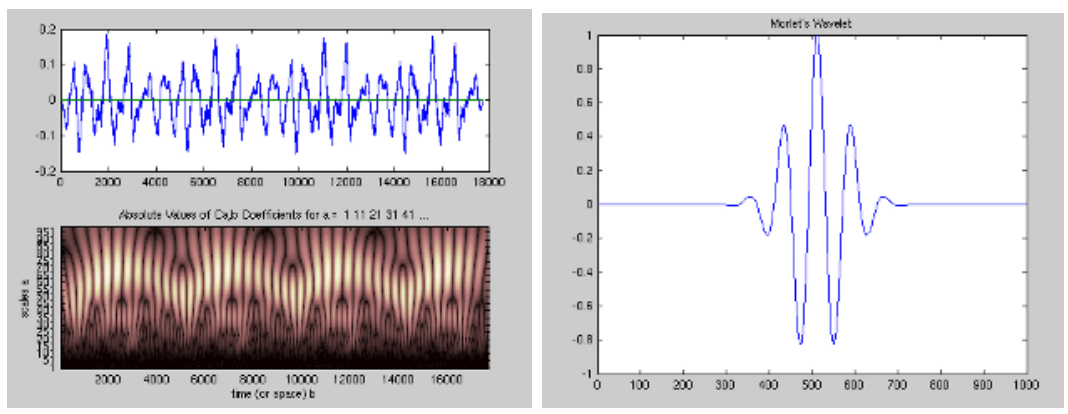

Fig. 2. Wavelet coefficients for a sound register and Morlet's mother wavelet

\section{Feature Extraction}

Continuous wavelet transform gives frequency and temporal information but, at the same time, it increases the processed data dimensionality. Hence, from a temporal signal in $\Re^{2}$ (time and intensity) a new signal in $\Re^{3}$ (time, scale and intensity) is obtained (figure 2). In order to process the information in a reasonable time it is necessary to restrict this processing to a small set of scales that represent the differences among the signals acquired at different locations.

For decades, principal component analysis (PCA) has been widely used to decorrelate spectral bands for reducing dimensionality. It is a useful technique if the spectral class structure of the transformed data is distributed along the first axes. This process is time consuming but it is only needed at the training stage.

First, we analyze the autocorrelation function of the sound register (figure 3) in order to find the lowest repetitive cycle delimitated by two global maxima. Then, the number of samples that feed the ANN will be proportional to this cycle. 


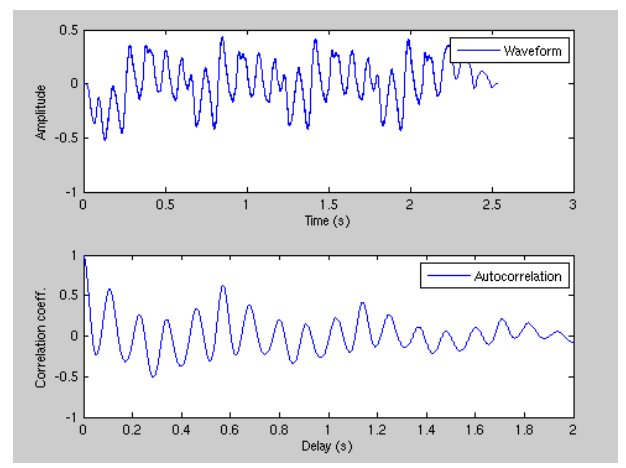

Fig. 3. A register of sound and its autocorrelation function

When the number of samples is already decided, the PCA is applied to determine how many data vectors are necessary for describing the information, that is, the so-called scale vectors. The data to calculate PCA are the vectors formed by all the scales for an specific sample. That implies to have 100-dimension vectors, that is, the CWT returns 100 scales for each sample. Figure 4 shows a Pareto diagram with the percentages of PCA results, and in the table 1 there are their numerical values. From this table we can observe that with 3 components it is explained the $75 \%$ of the information and with 5 components the $88 \%$. In order to reduce the amount of information to enter to the ANN, we have chosen 5 components, and despite to have less information in the data, the ANN yields very good results.

Table 1. The five main Principal Components of a sound register

\begin{tabular}{rrr}
\hline Principal C. & \% Information \% & Accumuled \\
\hline First & 42.84 & 42.84 \\
Second & 20.11 & 62.96 \\
Third & 12.00 & 74.97 \\
Fourth & 8.29 & 83.26 \\
Fifth & 4.73 & 87.99 \\
\hline
\end{tabular}

\section{Neural Networks}

Artificial neural networks are biologically-inspired systems, imitating the physical structure of nervous system with the intention of processing information in a parallel, distributed and adaptive way.

These systems can be considered as universal solvers for their generalization feature based on examples (this is the case of the supervised networks) or based 
on reinforcement. This behaviour permits to build a system to solve situations without having a deep knolwedge of the problem, and therefore, to consider the neural network systems as black boxes. These features make them specially useful as classifiers and interpolators (ie. feed-forward networks).

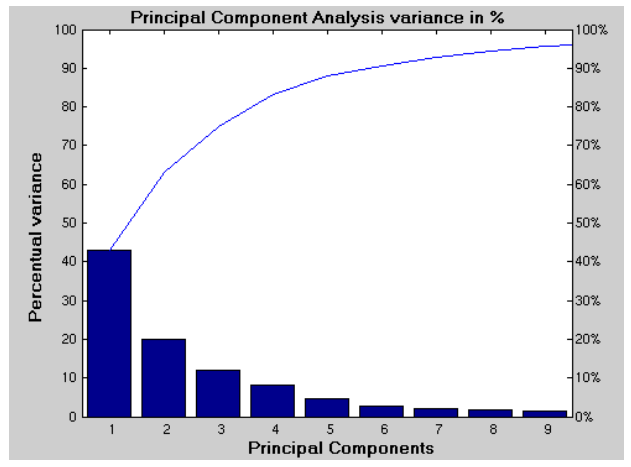

Fig. 4. Pareto diagram of Principal Components

The concept of black box has some drawbacks, such as the fact that it is not possible to extract the system behaviour since the networks is working correctly nor, consequently, the procedure to choose the more suitable network parameters (network structure, number of hidden layers, activation functions, ...).

Dynamic neural networks are divided in only feed-forward connections and feed-back connections (recurrent). Feed-forward dynamic networks (figure 5]) have a delayed tapped input and so, the output depends not only on the current input to the network, but also on the current or previous inputs. They are specially good systems for input sequences.

In the first tests, we realized about the importance of the tapped delay length, because the larger the amount of samples the lower the probability of resemblance with another sound registers. The ANN configuration is, specifically, 20 previous samples. Then, the hidden layer is made up with one hundred neurons.

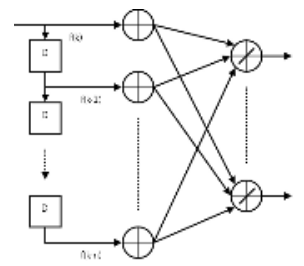

Fig. 5. Dynamic feedforward neuronal network 


\section{Experimental Results}

\subsection{Training Data Sets}

In order to check the reliability of the method proposed in this paper, we have obtained a set of signals in a room, as it can be seen in figure 7 As sound sources we have used three different industrial machines: milling machine, electric drill and an industrial drill. Each sound source has been placed in three different locations inside the room: at the center, on edge and in one corner of the room. These locations are marked with one crosses in the figure. For each sound source and for each location we have acquired twenty signals in specific locations, where its $\mathrm{x}-\mathrm{y}$ spatial coordinate is perfectly known. Each signal is approximately recorded for two seconds at $8 \mathrm{kHz}$ frequency sampling (more than 20,000 samples/signal). For each signal the CWT is calculated, obtained as many columns of scales as samples. Since the number of scales is approximately 100, the PCA reduces it to five scales. The input of the neural network is a vector with these five components (with its $\mathrm{x}-\mathrm{y}$ coordinates for training, and without them for classification). Furthermore, the number of input vectors for each signal is equal to the number of samples. This huge amount of data generates the same number of outputs, with are depicted in figure 6 . In this figure, it can be seen some clouds of points around a central points which is the training example, numbered in rows and columns as shown in figure 7, together with the sample number. For each set of twenty sound register, we use 18 registers for training and 2 registers for validation (crossed validation). In summary, we have obtained 180 signals that will be used to train three separated neural networks, with the consequent amount of information explained above.

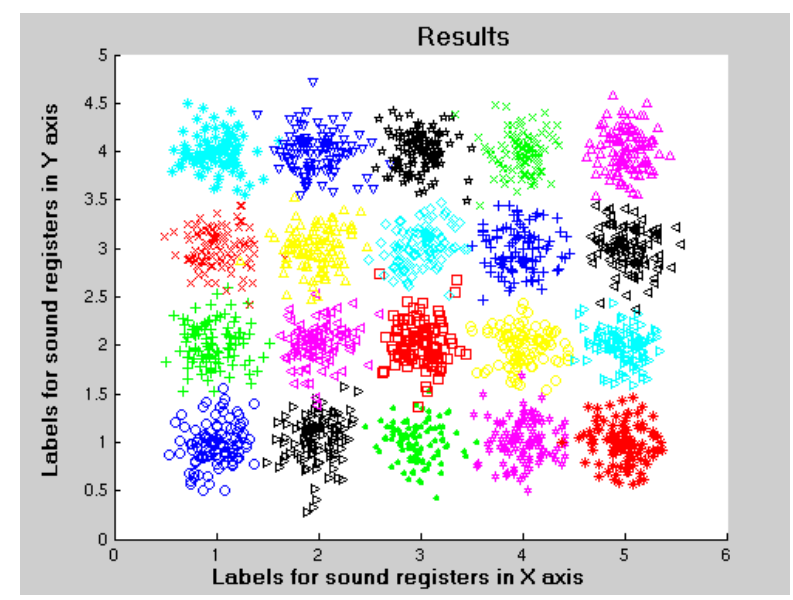

Fig. 6. Network output: $x-y$ localization for the sound registers. The axes indicate the label shown in figure 7 


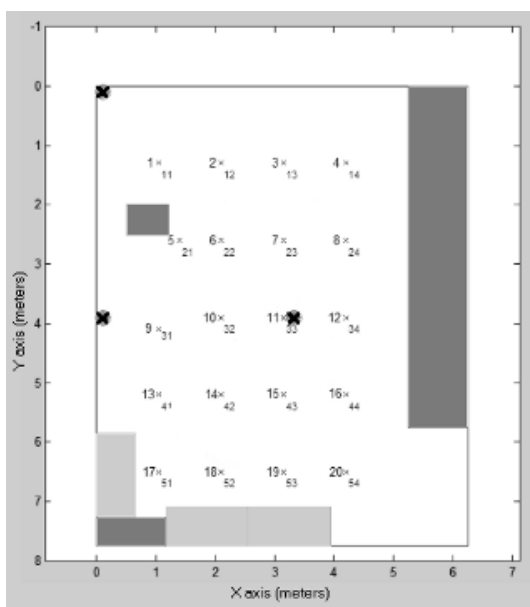

Fig. 7. Localization map of sounds registers and sound sources (big crosses)

Table 2. Classification ratio for training and validation with the milling machine in a corner of the room

\begin{tabular}{lc|cc}
\hline \multicolumn{2}{r|}{ validation } & \multicolumn{2}{c}{ training validation } \\
\multicolumn{2}{r|}{ sound registers } & $\%$ & $\%$ \\
\hline $1 / 1$ & $1 / 4$ & 77.2 & 62.1 \\
$1 / 2$ & $3 / 4$ & 76.2 & 65.1 \\
$1 / 3$ & $5 / 4$ & 81.1 & 64.2 \\
$2 / 1$ & $4 / 3$ & 72.5 & 69.4 \\
$2 / 2$ & $4 / 3$ & 76.1 & 71.5 \\
$2 / 2$ & $4 / 1$ & 83.7 & 68.8 \\
$2 / 3$ & $5 / 3$ & 79.8 & 78.0 \\
$2 / 3$ & $4 / 1$ & 81.3 & 72.1 \\
$3 / 1$ & $2 / 4$ & 74.1 & 73.6 \\
$3 / 2$ & $3 / 3$ & 86.2 & 45.1 \\
$3 / 2$ & $3 / 4$ & 80.5 & 63.0 \\
$3 / 3$ & $4 / 1$ & 83.1 & 69.5 \\
$3 / 4$ & $4 / 2$ & 76.0 & 61.2 \\
$4 / 2$ & $2 / 2$ & 84.5 & 79.5 \\
$4 / 2$ & $5 / 4$ & 84.9 & 66.7 \\
$4 / 3$ & $5 / 1$ & 73.7 & 71.6 \\
$4 / 4$ & $4 / 1$ & 87.1 & 66.5 \\
$5 / 2$ & $3 / 4$ & 82.6 & 71.3 \\
$5 / 3$ & $3 / 3$ & 79.9 & 61.2 \\
$5 / 4$ & $3 / 4$ & 86.0 & 81.3 \\
\hline \multicolumn{3}{r|}{}
\end{tabular}




\subsection{Results}

The results are slightly different depending on the localization and the source of sound. Table 2 shows some of these results, concretely obtained with a milling machine located in a corner of the room. The neural network have been trained for giving two coordinates $(\mathrm{x}, \mathrm{y})$ of a spatial 2D position. Since the output layer transfer function is linear, the $\mathrm{x}-\mathrm{y}$ output from the network will be considered classified within the area of the closest sample in the training stage. Table 2 shows the classification results. Each row in the table corresponds to the classification rate for a pair of samples used as crossed validation. The column "validation" means the average success in the classification of a specific sound register when it is used as a validation sample, whereas the column "training" means the average success in the classification of the eighteen remaining samples in the training stage.

\section{Conclusions}

In this work, a non-speech-based localization method is presented. This method uses the continuous wavelet transform (CWT) to capture frequency-time information of sound which, after a dimensionality data reduction process, feeds a dynamic neural network. The system is trained with samples with known $\mathrm{x}-\mathrm{y}$ position which have been acquired from 3 sound sources in three different sites in a room. The training process is relatively slow as well as the PCA analysis but this is an off-line process. The results demonstrate that the ANN localizes the new sound registers with a relative small error.

\section{References}

1. Bolea, Y., Grau, A., Sanfeliu, A.: Non-speech sound feature extraction based on model identification for robot navigation. CIARP 2003, LNCS 2905, pp. 221-228, 2003.

2. Bolea, Y., Grau, A., Pelissier, A., Sanfeliu, A.: Structural Pattern Recognition for Industrial Machine Sounds based on Frequency Spectrum Analysis. CIARP 2004, LNCS 3287, pp. 287-295, 2004.

3. Luo, H., Zeng, H., Hu, L., Hu, X., Zhou, Z.: Application of Artificial Neural Network in Laser Welding Defect Diagnosis. Journal of Materials Processing Technology vol. 170, pp. 403-411, 2005.

4. Huang, J., Supaongprpa, T., Terakura, I., Wang, F., Ohnishi, N., Sugie, N.: A modelbased sound localization system and its application to robot navigation Robotics and Autonomous Systems vol. 27, pp. 199-209, 1999.

5. Cowling, M., Sitte, R.: Comparison of techniques for enviromental sound recognition. Pattern Recognition Letters vol. 24, pp. 2895-2907, 2003.

6. Orozco-García, J., Reyes García, Carlos A.: A study on the Recognition of Patterns of Infant Cry for the Identification of Deafness in Just Born Babies with neural Networks. CIARP 2003, LNCS 2905, pp. 348-349, 2003. 